\title{
Minimal intervention dentistry II: part 6. Microscope and microsurgical techniques in periodontics
}

IN BRIEF

- Examines the use of the microscope in periodontics.

- Highlights the important of microsurgical techniques in achieving a successful and aesthetic result.

\author{
Y. Sitbon ${ }^{* 1}$ and T. Attathom ${ }^{2}$
}

Different aspects of treatment for periodontal diseases or gingival problems require rigorous diagnostics. Magnification tools and microsurgical instruments, combined with minimally invasive techniques can provide the best solutions in such cases. Relevance of treatments, duration of healing, reduction of pain and post-operative scarring have the potential to be improved for patients through such techniques. This article presents an overview of the use of microscopy in periodontics, still in the early stages of development.

The contribution of the operating microscope to dentistry in general has been described in another article by the same authors elsewhere in this series. The need to magnify poorly lit and extremely small operating fields, the necessity of allowing the utmost precision during our procedures, both in

\section{MINIMAL INTERVENTION DENTISTRY II \\ 1. Contribution of the operating microscope to dentistry \\ 2. Management of caries and periodontal risks in general dental practice \\ 3. Management of non-cavitated (initial) occlusal caries lesions - non-invasive approaches through remineralisation and therapeutic sealants \\ 4. Minimal intervention techniques of preparation and adhesive restorations. The contribution of the sono-abrasive techniques \\ 5. Ultra-conservative approach to the treatment of erosive and abrasive lesions \\ 6. Microscope and microsurgical techniques in periodontics \\ 7. Minimal intervention in cariology: the role of glass-ionomer cements in the preservation of tooth structures against caries \\ 8. Biotherapies for the dental pulp \\ This paper is adapted from: Sitbon Y, Attathom T. Microscope et techniques microchirurgicales en parodontologie. Réalités Cliniques 2012; 23: 223-233.}

College of Dentistry, University of Montreal, Canada: ${ }^{2}$ College of Dentistry, University Chulalongkorn, Bangkok, Thailand

${ }^{*}$ Correspondence to: Yves Sitbon

Email:Yves_sitbon@yahoo.fr

\section{Refereed Paper}

Accepted 15 November 2013

DOI: 10.1038/sj.bdj.2014.356

${ }^{\circ}$ British Dental Journal 2014; 216: 503-509 order not to damage surrounding structures and to avoid creating bacterial retention niches, have been put forward in a global dental context. The ergonomic benefits of working under microscope magnification have also been mentioned in another article.

In spite of those obvious advantages, the use of the operating microscope in dentistry is not yet widely spread. At the most, it has started to develop in a more systematic way in endodontics, and even then, mostly within practices specialised in such treatments. The main reasons for such disinterest in a technique, however, fiercely defended by its users, probably resides in the acquisition cost of the microscope, the rather steep learning curve of the technique and most likely, the lack of information within the profession. The purpose of this article is to present a few advantages, specific to periodontology, to the greater use of magnification and microsurgical techniques. The reasons why one should favour a microscope over loupes has been described in the article on the contribution of the microscope to dentistry in general and will not be repeated here. However, in a few specific cases loupes can indeed be a better choice. These cases will be mentioned in this article, and we will address possible uses of the microscope for periodontal disease treatment itself, and then for mucogingival surgery. A few clinical cases will complete this overview of contribution of microscopy in periodontics.

\section{CONTRIBUTION TO PERIODONTAL DISEASE TREATMENT}

Periodontal disease can be defined as an infectious, bacterial disease, resulting in an attack of tooth-supporting structures: bone, gums, cement and the ligament system that anchors the tooth to the bone, including the epithelial attachment. This disease presents various degrees of severity, rate of progression, and response to treatments. Addressing those items, however, is beyond the scope of this article; but it is worth pointing out that regardless of the considerable progress achieved over the last decades in periodontal treatment, a few patients see their condition deteriorate, in spite of their efforts to maintain proper oral hygiene, and the efforts of their practitioner to stop the disease. Moreover, in certain cases, it is precisely those treatments, sometimes too aggressive, that induce other problems, replacing or worsening the initial insult. Gingival recession with unsightly root exposure and increased caries risk, loss of cement with dentinal exposure and associated hypersensitivity, loss of papillae, or clinical crown lengthening with cosmetic prejudice, are just a few examples of those problems.

It seems therefore that improvement of either the diagnostics tools, or the quality of the dentist's procedures, or even better, both, could increase the success rate of the treatment, and at the same time reduce complications, especially of iatrogenic nature. This would be a first step toward minimally interventionist and minimally invasive dentistry.

This article will focus on improvements the microscope can offer. It seems nevertheless judicious to stress that the microscope is only a magnification tool. It is neither a technique in itself, nor an instrument that allows a specific technical act. It will assist the operator in carrying out particularly sensitive procedures otherwise difficult if not impossible to achieve. Improvement of vision also allows the operator to see tiny 
details that could potentially have been left undetected, and thus, increases its diagnostic capacity.

\section{Contribution of the microscope during the diagnostics phase}

The most common means used to diagnose periodontal disease is visual examination, assisted by a periodontal probe and radiographs. ${ }^{1,2}$ The periodontal probe gives a quantified reading of periodontal tissue damage, and the radiographs allow visualisation of some of the structures not visible by direct vision, especially the interproximal bone.

Probing is done with a precision of about one half of a millimetre. This degree of precision is easily obtained without a magnification tool. However, magnification will offer a more comfortable and faster reading of the probe markings and a better illumination of the operating field if a light generator is added. But moving from one tooth to another requires a great depth of field and high magnification would go against that necessity. Besides, the operator requires a great freedom of movement (to progress from one quadrant or one side of the mouth to the other) and using a microscope is probably not useful, if not counter-productive. In that case, loupes are best suited for |those tasks.

As for the radiographic examination, here again loupes will allow a sufficiently accurate reading of the information carried by the film. Moreover, radiographs are becoming more and more digital in nature, and it is possible to zoom in as desired on the particular zones of interest. In this case, it is the resolution of the captor, and that of the screen, that will be the limiting factor for the precision of the diagnosis.

Therefore, it seems that a microscope does not significantly contribute to the initial diagnostics phase of periodontal disease. Nevertheless, during complementary visual examination to investigate possible local causes of greater severity or resistance of the disease to treatment, the microscope can be a helpful tool. For instance, all its potential comes in play when trying to visualise cracks or root fractures that could lead to a localised periodontal defect. It also allows a much more refined evaluation of the margins of a dental restoration, and to determine whether or not these margins could be a significant bacterial niche. After initial periodontal therapy, the dental operating microscope (DOM) allows the operator to track more efficiently any remaining irritating elements such as a spicule of calculus, or an enamel pearl, that could explain the loss, or persistence of loss, of epithelial attachment in a particular zone.

\section{Contribution of the microscope during initial treatment phase}

In a similar way, it seems reasonable to state that scaling-surfacing, one of the major components of periodontal disease treatment, ${ }^{4,5}$ can be very comfortably achieved using loupes only. The use of the microscope and its higher magnification and illumination could then be limited to assess the result of the procedure in more severely attacked, or more critical zones, such as furcations, and possibly to complete the work in those places.

Nevertheless, persistence of dental plaque and calculus on root surfaces after scaling-surfacing procedures has often been described in scientific literature. ${ }^{6,7}$ In parallel, numerous studies have shown that this procedure presents a better deposit-removal if done with a flap exposure. ${ }^{8-11}$ This improvement has been associated with better accessibility to the surfaces, but mostly better visibility. It thus appears legitimate to infer that improving vision further, by means of a more powerful magnification tool, would favour an even better outcome., ${ }^{3,12,13}$ Moreover, if vision and deposit-removal were improved at the initial phase of treatment, the probability of needing subsequent surgical treatments would be reduced, and with it all the disadvantages linked to surgical procedures.

It is thus the dentist's decision to perform the initial scaling-surfacing phase under microscope magnification, depending on the severity of the disease, its resistance to previous treatments if any, and the difficulty to visually access the site. ${ }^{14}$ Magnification will naturally increase the duration of the procedure, and as such the doctor must carefully evaluate the cost/benefit ratio for the patient.

We could modulate this statement by saying that rather than the need for magnification, it is mainly the need for the procedure itself that needs to be carefully assessed. It has been reported that many of the procedures accepted as standard treatment actually lack proper scientific support to be fully recommended, and that at least their frequency should be re-evaluated. ${ }^{15,16}$

If such treatment is indeed needed, then we ought to carry it out with utmost possible control to avoid any destructive effect, and thus, magnification should be a standard of care. For instance, scalingsurfacing, whether with or without flap, is more technique-sensitive than it might seem. Whichever technique is used (manual, sonic, ultrasonic, rotating instruments) can have damaging effects on the structures. ${ }^{17}$ Performing the procedure under high magnification allows instant feedback on the effect of the treatment on the elements that we want to remove, as well as on the elements that we want to keep intact. This also contributes to a real learning experience on how to use the instruments in the most efficient and less traumatic possible way.

\section{Eradication of local factors}

Eradication of dental plaque and calculus deposit is not the only objective of the initial treatment phase. Actually, elimination of all potential bacterial niches that could potentially induce or maintain a periodontal problem is part of it as well. Re-contouring a restoration with poor outline, especially in the interdental spaces, is an example of such an objective. But this is by no means an easy task, especially if one wants to stay away from the surrounding healthy structures. For instance, using alternating-motion hand pieces, such as the EVA, helps to get closer to that goal, ${ }^{18,19}$ but such tools can express their full potential only when used in conjunction with appropriate magnification. Indeed, the human eye has an acuity of about 150-200 $\mu \mathrm{m}$. Hence, defects of smaller size are left unseen, but they would still represent niches of significant size at a bacterial scale. And damage to surrounding structures (epithelial attachment and cement predominantly), unless major, would not be perceived by the operator, and therefore, probably not be prevented in his practice.

\section{Contribution of the microscope in the surgical phase of the treatment}

If, in spite of all the efforts spent so far, initial treatment phase would prove insufficient to stop the progression of the disease, the practitioner could opt for a surgical treatment, aiming at better exposure of the surfaces to clean and also at reconstructing a positive architecture, and better cleansability by pocket elimination. We already addressed potential advantages of using a microscope for the surfacing-scaling component of those procedures, but not those for the surgical phase of opening and closing the site (flap and sutures). These advantages are the same as for mucogingival surgeries and will be described in the following section.

\section{CONTRIBUTION OF THE MICROSCOPE AND MICROSURGICAL TECHNIQUES TO PLASTIC MUCOGINGIVAL SURGERY}

Microsurgery has been defined as the refinement of conventional surgical techniques by using magnification tools and specific instruments. ${ }^{20}$ The paternity of micro-mucogingival surgery can largely be attributed to Dr Shanelec. As a pioneer for the development of these techniques, and under his impetus and his guidance, other practitioners 


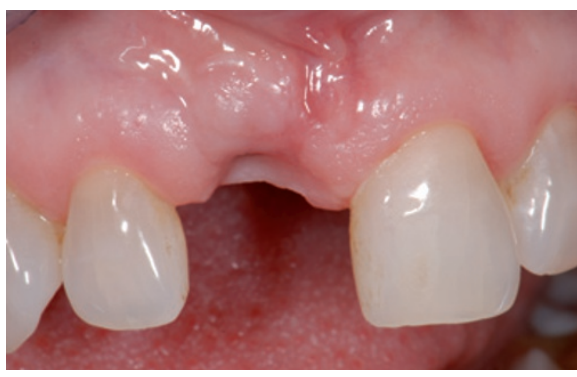

Fig. 1 An indelicate handling of the soft tissues during implant placement has resulted in unsightly scars

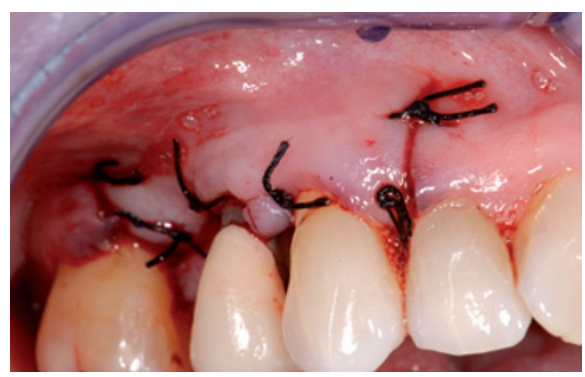

Fig. 2 Conventional surgical techniques often show trauma to the tissues and insufficient closure of the incisions lines, leading to healing by second intention, which is slower and more likely to generate scar tissue

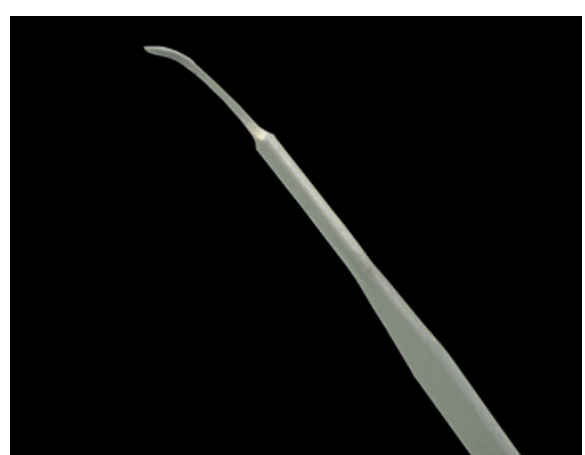

Fig. 3 An IBN6900 microblade (Hartzell and Sons) is curved into a J-shape at its end, to allow its passage underneath the papilla to dissect it from its bony support

embraced this technique, establishing a toehold among those in the profession. The periodontium is a complex structure, presenting extremely delicate zones such as the epithelial attachment, the marginal gingiva, and the interdental papillae. The bony support itself is prone to damage just by the mere fact of exposing it during a surgical procedure. Yet patients' requirements have increased, especially when it comes to aesthetics, meaning mucogingival surgical techniques are more and more demanding for the psychomotor skills of the doctor and represent a real challenge. If healing of the soft tissues of the mouth is generally forgiving, compared to dermis, for example, it is nevertheless true that an inadequate surgical gesture can lead to unsightly scarring (Fig. 1). When tissues are observed under microscope

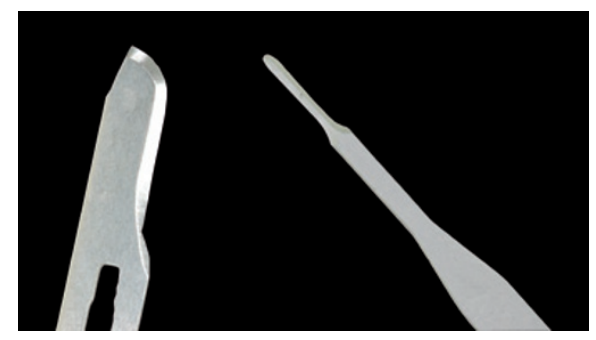

Fig. 4 Comparison between a $15 \mathrm{C}$ blade and an IBN6900 blade, highlighting the difference of size
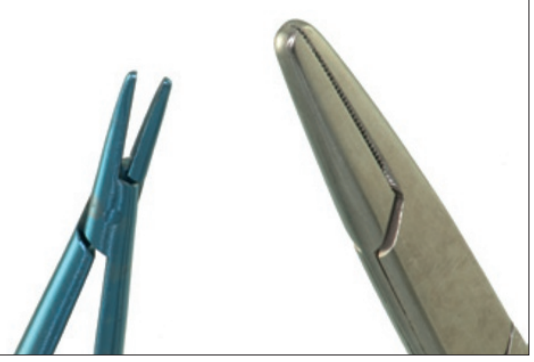

Fig. 5 Comparison between standard suturing forceps and microsurgical forceps (ING1300S, Hartzell and Sons). The conventional forceps would not allow the needle to be grabbed without distorting it or breaking it, making it useless

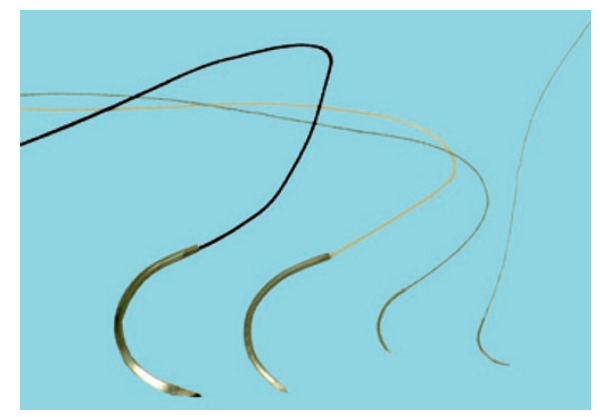

Fig. 6 Four different sutures are shown to highlight the difference of size of the needles and the threads. From left to right: Ethicon 3-0, silk multifilament, $22 \mathrm{~mm}, 3 / 8$ reverse cutting needle; Ethicon 4-0, coated Vicryl, $19 \mathrm{~mm}, 3 / 8$ reverse cutting needle; Ethicon 7-0, nylon monofilament, $8 \mathrm{~mm}, 3 / 8$ reverse cutting needle; Ethicon 9-0, nylon monofilament, $5 \mathrm{~mm}, 3 / 8$ tapered needle

after a conventional surgical procedure, it is amazing to witness the gaping suturing and the damage inflicted to these tissues, caused from laceration and crushing (Fig. 2). ${ }^{15}$

Thus, an unrefined surgical technique can lead to aesthetic and/or functional harm. Many factors influence such outcome: the extent of the surgical field, the type of instruments used, but also the dentist's technique. If a microsurgical approach considerably improves the first two elements, the technique of the operator can be either improved or reduced by this approach, as it is extremely demanding in terms of control. The results will be in conformity with the efforts the practitioner will spend on mastering those techniques.

\section{Microsurgical instruments}

Microsurgical techniques are hardly feasible without a magnification tool given the delicateness of the tissues being manipulated. For the same reasons, they are best carried out with specific instruments and sutures (most often imported from ophthalmic surgery), the small size of which increases the need for magnification even more. Using conventional instruments is not really an option; indeed, their size would imply a larger surgical access, which goes against the whole concept and philosophy of microsurgery. Besides, specific gestures are just not achievable without the proper instrument. For instance, during papillae augmentation procedures (see clinical cases 1 and 2), the dentist needs to dissect the papilla within the embrasure. This is best done with an ophthalmic blade, curved in a 'J' shape (Fig. 3), which can be run under the papilla to separate it from the underlying bone support, progressing in the narrow space of the dental embrasure. A traditional blade, such as a $15 \mathrm{C}$ blade, besides being oversized (Fig. 4), cannot be bent in that J-shape, a shape that is necessary to separate the papilla from the bone without severing it altogether. By the same token, only very small periosteal elevators can raise a mini-flap without tearing it. Another example of a micro-instrument is the specific suturing forceps (Fig. 5). Indeed, the needles of microsutures are so small that the mere action of grabbing them with a regular suture forceps would deform them to a point that they could not be used anymore, or even possibly break them (Fig. 6).

These microsurgical instruments must comply with a very strict bill of specifications. They have to be light to prevent hand fatigue, but still be rigid enough to maintain proper stability. They need to be long enough to rest on the saddle formed by the thumb and the index, and if possible have round handles to allow rotational movements. They must be non-reflective to avoid blinding the operator (especially with the high-intensity light of the microscope), and should be nonmagnetic. Forceps need to be machined to a very high level of precision to allow a good grip on very small needle or very delicate tissue without damaging them, and still allow an easy opening-closing action. These extensive requirements result in higher costs and the need for more careful manipulation during cleaning and sterilisation procedures.

\section{Microsutures}

Usually, sutures of size 7-0 and smaller are considered as microsutures. The suture material is extremely thin and not easily seen by the naked eye (Fig. 6). Knots are not done in a conventional way, securing them is down to visual control only. Chiefly, in order to 


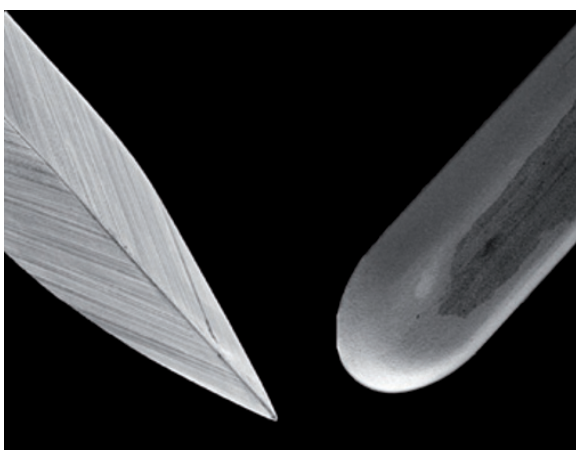

Fig. 7 Scanning electronic microscope view of the edge of a $15 \mathrm{C}$ blade and a crescent ophthalmic blade (IB940011, Hartzell and Sons). The $15 \mathrm{C}$ blade is machined, presenting a rougher surface than the ophthalmic blade, obtained by electrolytic process

provide enough slack to allow tissue oedema (without which, tissue would tear or become ischemic at the entrance and the exit points of the suture), the knots are only loosely tightened, making tactile control impossible. Also, given the thinness of the suture material, relying upon tactile control would most certainly result in breaking the suture, or worse, tearing the tissue. Using the microscope, or at least high magnification loupes (with all their associated disadvantages), is virtually mandatory to provide the required visual control.

If those difficulties can seem crippling in the first place, there is still a major advantage to using microsutures, which is the ability to place the stitches closer to each another and thus obtain a better closure of the wound. This allows for a first-intention healing process, and consequently a reduction of healing time and risk of a scar. Last, but not least, they provide a much better comfort for the patient, the sutures being almost imperceptible. On the other hand, more stitches being necessary, procedure duration is increased.

Needles being smaller, they can be run several times in the papilla without severing it or compromising its blood supply. Also, with the chord of the needle (distance in a straight line between its tip and its crimping) being short, the needle can be run in very small spaces, as when closing the short segment, perpendicular to the sulcus, or releasing incisions, (design being thought as more favourable in terms of healing). ${ }^{22}$ A longer needle would require a two-step passage, with entry and exit points not perpendicular to the tissue. Moreover, a larger needle would increase the risk of compromising the blood supply or of laceration of the tissue.

\section{Rationale in favour of microsurgical techniques}

Thanks to the simultaneous use of microsurgical techniques, smaller instruments, microsutures, as well as a microscope allowing a
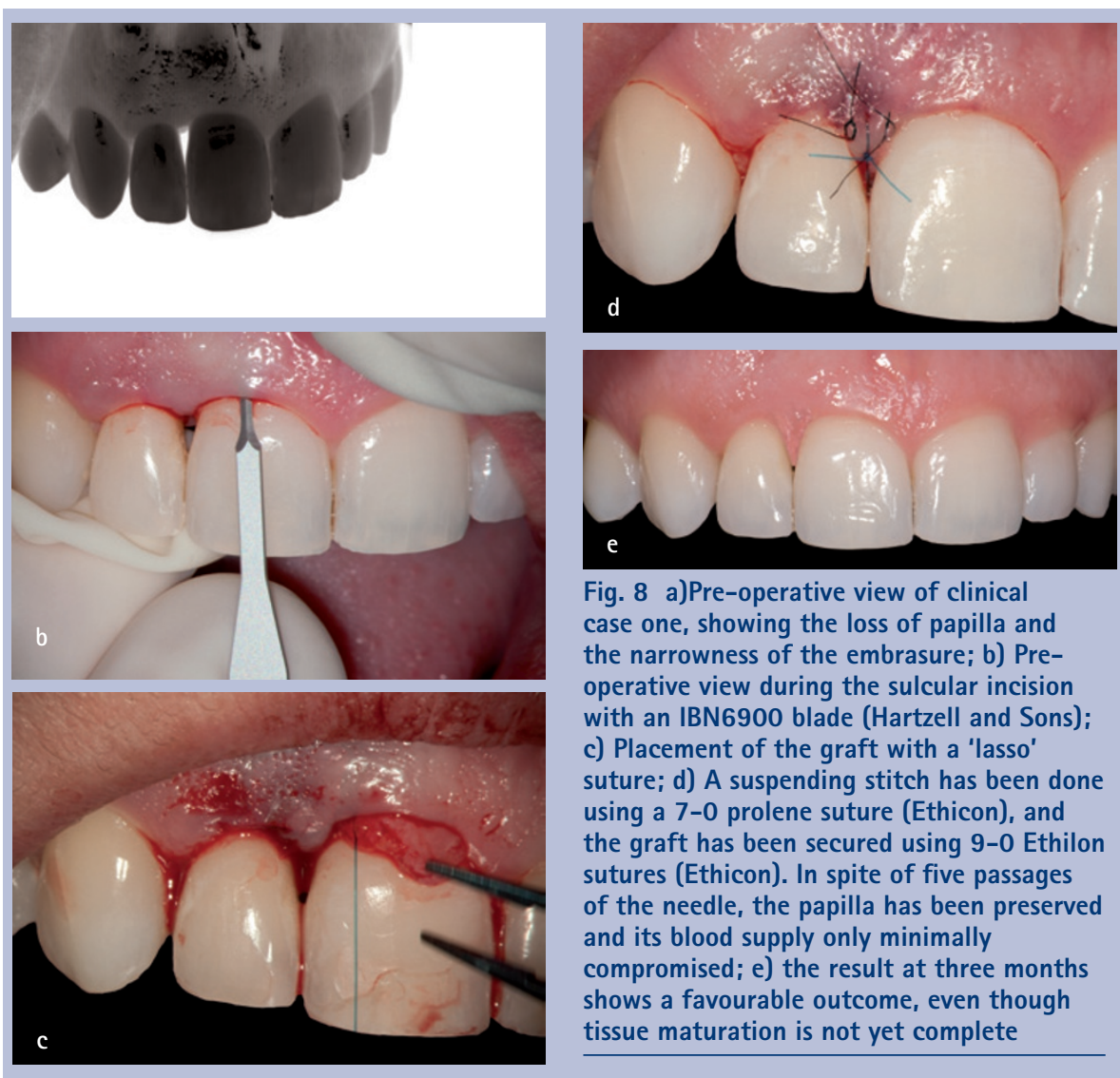

Fig. 8 a)Pre-operative view of clinical case one, showing the loss of papilla and the narrowness of the embrasure; b) Preoperative view during the sulcular incision with an IBN6900 blade (Hartzell and Sons); c) Placement of the graft with a 'lasso' suture; d) A suspending stitch has been done using a 7-0 prolene suture (Ethicon), and the graft has been secured using 9-0 Ethilon sutures (Ethicon). In spite of five passages of the needle, the papilla has been preserved and its blood supply only minimally compromised; e) the result at three months shows a favourable outcome, even though tissue maturation is not yet complete

good control of all those elements, patients and doctors alike can enjoy a greater comfort and achieve an optimal result. The rationale supporting the use of microsurgical techniques, besides the advantages mentioned above, is based on the following observations:

- The larger the spread of the soft and hard tissues included in the manipulation, the greater the inflammation, the oedema, the postoperative pain, and the healing time. Limiting the extent of the surgical site is thus an obvious factor for reduced morbidity. Being very small, microsurgical instruments allow, when indicated, to considerably reduce the size of the operating field

- The more the blood supply to the tissues handled is compromised, the greater the risk of necrotic zones, with related aesthetics and functional consequences potentially following

- If the design of the flap is essential to prevent compromising blood supply ${ }^{22,23}$ using a microscope allows to avoid certain incisions altogether, contributing to a better blood perfusion of the tissue. For instance, when harvesting a connective tissue graft from the palate, magnification makes it technically easier to proceed with a unique incision line, running parallel to the cervix of the teeth, avoiding releasing incisions that would deny the flap from one source of its blood supply. Similarly, immediate implant placement, directly following tooth extraction, even combined with a thickening of soft tissue with a connective tissue graft, can be done with no releasing incision if visibility is adequate, which is more likely to be the case under magnification via a microscope and its adequate illumination. This represents an undeniable advantage to maintain optimal blood flow in the area, but also for papillae preservation, and to maintain the buccal cortical plate, which no longer needs to be exposed. Moreover, the risk of scars is eliminated and it has been shown that microsurgical techniques can lead to a faster revascularisation of the grafts ${ }^{24}$

- Tissues suffer more from crushing than incisions or puncture. The edginess of ophthalmic surgical blades is obtained by electrolytic process, rather than machined..$^{12}$ The blade edges are therefore sharper, and the surface texture of the blade smoother, resulting in a perfect incision, with the least possible amount of trauma (Fig. 7). Also, using forceps with microscopic teeth allows the operator to delicately seize soft tissues and reposition them, rather than using blunt instruments that are more likely to crush those fragile tissues and hence potentially induce necrosis 

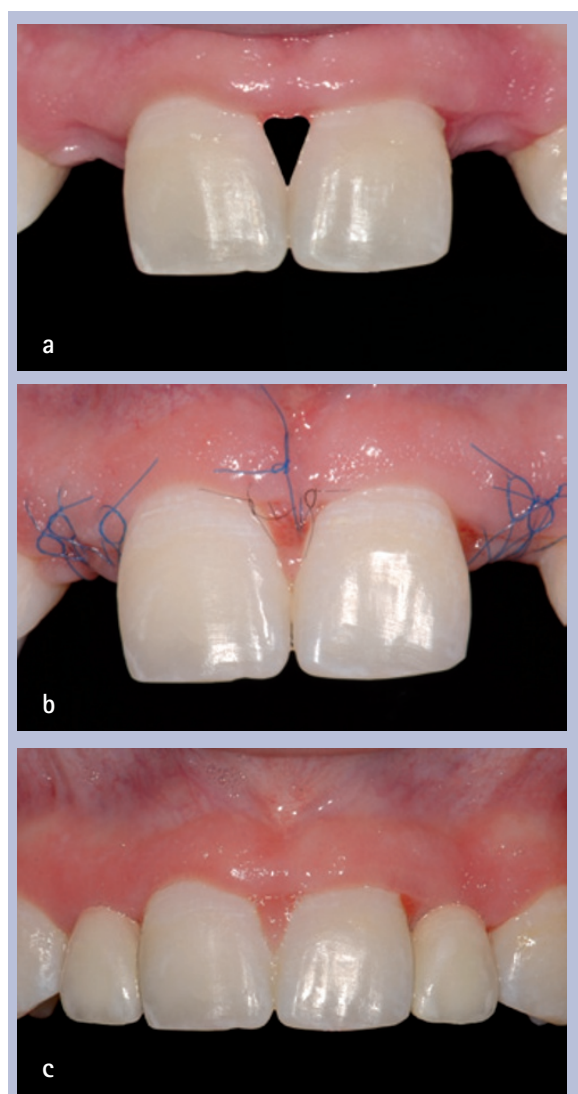

Fig. 9 a) Pre-operative view of case two, with congenitally missing laterals and loss of papilla. In this case, the embrasure is wide, which facilitates the procedure; $b$ ) Post-operative view at five days, before removal of sutures. Implant sites have been closed with 7-0 prolene suture (Ethicon), and the papilla has been sutured, folded on itself, using a couple of 9-0 ethilon (Ethicon) stitches. The mediocre quality of one of the stitches can be readily noticed, and has been immediately penalized by a localized necrosis of the tissue; c) Result at three months, after final restorations have been placed. A scar can be seen at the base of the papilla, corresponding to the necrotic zone, mentioned above

- Healing by first intention is more desirable than by second intention; inflammation, oedema, post-operative pain, healing time, are reduced, as well as the risk of scarring. The practitioner should then favour a perfect closure of the wound. As mentioned earlier, the small size of microsutures allows him or her to place the stitches closer, providing a perfect coaptation of the edges of the incision, using magnification to control such coaptation

- Risk of scar, pre and post-operative pain, healing time, discomfort from the stitches and visibility of the later are all reduced; thus acceptance of treatment by patients is greatly improved

- Reduced bleeding, better vision, more comfortable posture, better results, satisfaction of a well-controlled procedure, all contribute to the improved environment the dentist will be practising in.

\section{DISCUSSION}

More and more reports point out optimised results when using magnification during periodontal treatments, especially mucogingival plastic surgery. Unfortunately, these techniques are still not confidently used, not only due to the steep learning curve, but also because dentists are not aware of them.

Simultaneously, the lack of serious scientific research highlighting the actual advantages for the patients to be treated with these techniques has probably led to the mistrust of many periodontists when one suggests the improvements offered by the use of a microscope. Most of the articles published are anecdotal in nature and the few research articles that exist suffer major bias. ${ }^{29-31}$

Microsurgical techniques require a long, steep, learning curve, and it is unlikely that practitioners will be willing to devote so much time and effort to learn them if they are not convinced upfront of the superiority of these techniques. One can reasonably infer that anyone trying to publish on this particular topic has a favourable prejudice that will bias any research protocol.

\section{CLINICAL CASES}

A few examples of clinical cases of micromucogingival surgery are presented to illustrate the possibilities these techniques can offer to the operator.

\section{Case one}

The patient is 35 years old and lost the papilla between 12 and 11, after an overzealous scaling-surfacing, with a class $3^{4}$ loss, according to Nordland-Tarnow classification (Fig. 8a). ${ }^{33}$

Otherwise the patient presents a periodontium in good health, excellent oral hygiene and largely exposes her gum when she smiles.

After having considered alternate treatments (attempts to regenerate the papilla by repeated curettage, ${ }^{34}$ closing the space with composite resin, or simple abstention), the patient elected for a sub-papilla connective tissue graft.

The case is especially difficult given the very narrow embrasure and inverted architecture. The patient was informed about possible failure, but wished nevertheless to proceed with the microsurgical-grafting attempt. She also presents on the lingual of the upper incisors and canines a bonded metal wire serving as an orthodontic retainer, which complicates the procedure further, but will also allow for the lifting and anchoring of the papilla in order to provide the necessary space for the graft.

After freeing it from its underlying bone support using an ophthalmic blade (Hartzell and Sons, IBN6900), and after its mobilisation (Fig. 8b), the papilla is lifted away from the bony support using a 7-0 suture (Ethicon, Prolene, monofilament polypropylene, P-6, $8 \mathrm{~mm}, 3 / 8$ reverse cutting needle), looping around the metal wire retainer.

The connective graft, harvested from the palate, is tucked under the papilla (Fig. 8c) and secured with two stitches (9-0, Ethicon, Ethilon, monofilament nylon, BV130-4, $5 \mathrm{~mm}, 3 / 8$ needle). Using these microsutures has allowed five passages of the needles in the papilla, without severing it, or compromising too much its blood supply (Fig. 8d).

The result after three months shows a partial closure of the embrasure (class 1) (Fig. 8e), but it is reasonable to expect a complete closure within the few next months, provided oral hygiene procedures are not too aggressive.

\section{Clinical case two}

The patient is 19 years old, in good health, but has congenitally missing upper laterals that she wants to replace. She also presents a class $3^{4}$ loss of papilla between 11 and 12, following the resection of the labial frenum (Fig. 9a). After having discussed the different therapeutic options, decision was taken to place two implants and to reconstruct the papilla using microsurgical techniques, in spite of rather average oral hygiene.

Implants have been placed using both sulcular and mesio-distal crestal incisions, without releasing incisions, and with a gentle separation of the soft tissue from the cortical bone. The papilla has been reconstructed using a 'roll' technique. This technique consists in dissecting the papilla from its bony support, as described above, then to cut its palatal attachment, and finally to fold it on itself and suturing it in place using 9-0 and 7-0 sutures. The palatal zone heals by second intention. The implant sites have been closed using 7-0 sutures. Five days after the procedure sutures are ready to be removed. One can notice a mild necrosis at the papilla level, probably caused by a somewhat indelicate manipulation of the tissue (Fig. 9b). Three months later, the final restorations are placed on the implants. The aesthetics of the papilla is improved, even though the tissues are not mature yet, especially around the implants. It is worth noticing that where the small necrosis occurred at the base of the papilla, healing is not perfect, with a small scarring visible, emphasising the importance of the utmost delicacy when handling those fragile tissues (Fig. 9c). 


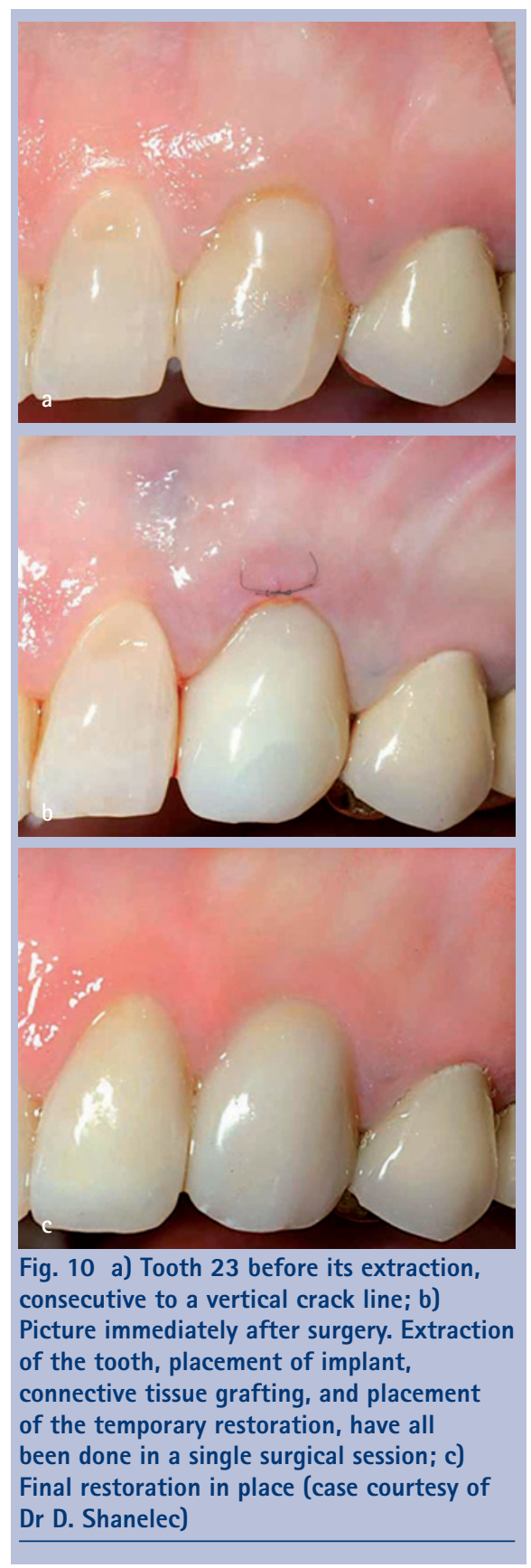

\section{Clinical case three}

This patient has benefitted from implant treatment, where atraumatic extraction of tooth 23, implant placement, connective tissue grafting and placement of the temporary crown have all been done in a single surgical phase, made possible by the control provided to the practitioner by the microscope (Figs 10a-c).

\section{Clinical case four}

This patient presents an important recession on tooth 13, and wishes to correct the defect for aesthetic reasons (Fig. 11a). A submerged connective graft, with coronal displacement of the flap has been done with minimal trauma to the tissue (Fig. 11b). A close-up view shows the perfect closure of the incision line (Fig. 11c). Similarly, the
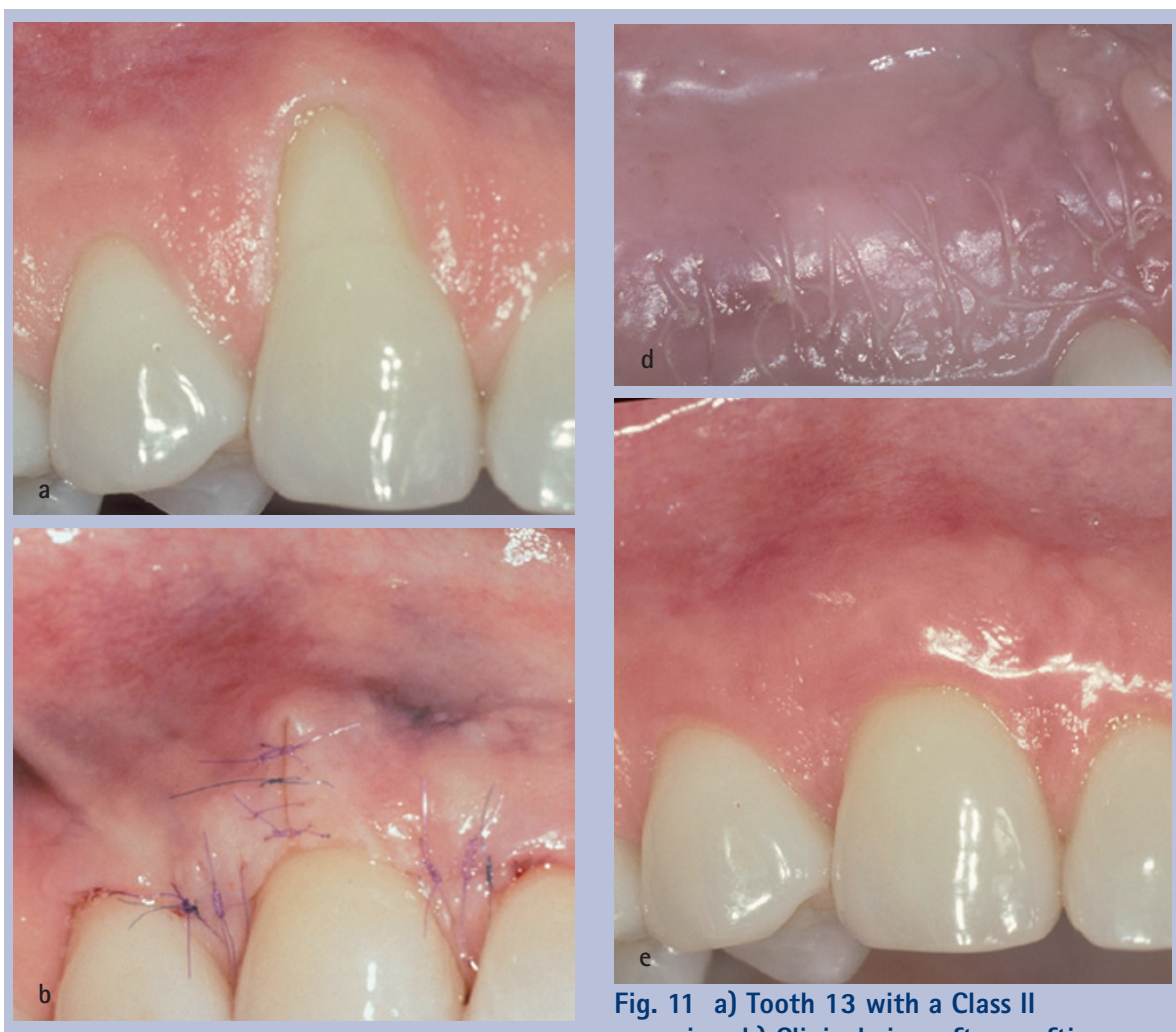

Fig. 11 a) Tooth 13 with a Class II recession; b) Clinical view after grafting, flap repositioning and microsuturing; c) $\mathrm{A}$ close-up view shows the excellence of the work, with a perfect incision line closure, allowing for a first intention healing; d) The palatal view shows the excellent tissue response at five days; the incision line is not visible anymore. It is worth noticing that the thin suture is not easily visible, especially when the thread is not of dark colour; e) The case finished, with a remarkable aesthetic outcome (case courtesy of Dr. D. Shanelec)

donor site healed very fast, thanks to a first intention healing of the site, also perfectly closed (Fig. 11d). The final results show a complete coverage of the root and a very beautiful aesthetic integration of the soft tissues, without any scars (Fig. 11e)

\section{CONCLUSIONS}

Even though scientific literature has not yet really shown an advantage to using microsurgical techniques, it is nonetheless true that all practitioners employing them are fierce advocates of their use. This enthusiasm, as well as the more and more frequent case reports, should encourage periodontists, specialists and non-specialists alike, to consider using these techniques and spend some time and efforts acquiring the necessary skills. Only then could they decide if microsurgery is a path they wish to follow. If the number of dentists using microsurgical techniques increases, clinical studies will be easier to implement, and will possibly bring more answers regarding the merits of this approach. This has actually been the case in endodontics, where it is under the sceptical eyes of their colleagues that Noah Chivian, and later Gary Carr, tried to promote the use of the microscope in their field. Since then, it has become a standard of care, and learning to work under microscope has been made mandatory in speciality programmes in North America ${ }^{32}$ In the meantime, microscope and microsurgical techniques certainly represent excellent ways to maintain our passion for a more and more demanding profession.

The authors would like to thank Claudie DamourTerrasson, publishing director of the Groupe Information Dentaire, Paris France, for the authorisation of translation and publication of the series in the $B D J$; Dr D. Shanelec for the photographs in Figures 10 and 11 included in clinical cases three and four; Mr Alvin X. Gee for editing the English manuscript; Dr Avijit Banerjee for his support.

1. Consensus report. Periodontal diseases: epidemiology and diagnosis. Ann Periodontol 1996; 1: 216-222. 
2. Armitage G C. Periodontal diseases: diagnosis. Ann Periodontol 1996; 1: 37-215.

3. Kwan J Y. Enhanced periodontal debridement with the use of micro ultrasonic, periodontal endoscopy. J Calif Dent Assoc 2005; 33: 241-248.

4. Lindhe J, Nyman S. Long-term maintenance of patients treated for advanced periodontal disease. J Clin Periodontol 1984; 11: 504-514

5. Lindhe J, Westfelt E, Nyman S, Socransky SS, Haffajee A D. Long-term effect of surgical/nonsurgical treatment of periodontal disease. J Clin Periodontol 1984; 11: 448-458.

6. Cobb C M. Non-surgical pocket therapy: mechanical. Ann Periodontol 1996; 1: 443-490.

7. Kepic T J, O'Leary T J, Kafrawy A H. Total calculus removal: an attainable objective? J Periodonto/ 1990; 61: 16-20.

8. Buchanan S A, Robertson P B. Calculus removal by scaling/root planing with and without surgical access. J Periodontol 1987; 58: 159-163.

9. Sherman P R, Hutchens L H, Jr., Jewson L G. The effectiveness of subgingival scaling and root planing. II. Clinical responses related to residual calculus. J Periodonto/ 1990; 61: 9-15.

10. Sherman PR, Hutchens L H Jr., Jewson L G, Moriarty $J$ M, Greco G W, McFall W T Jr. The effectiveness of subgingival scaling and root planning. I. Clinical detection of residual calculus. J Periodonto/ 1990; 61: 3-8.

11. Wylam J M, Mealey B L, Mills M P, Waldrop T C, Moskowicz D C. The clinical effectiveness of open versus closed scaling and root planing on multirooted teeth. J Periodontol 1993; 64: 1023-1028.

12. Belcher J M. A perspective on periodontal microsurgery. Int J Periodontics Restorative Dent 2001; 21: 191-196.
13. Hegde $R$, Sumanth $S$, Padhye A. Microscopeenhanced periodontal therapy: a review and report of four cases. J Contemp Dent Pract 2009; 10: E088-096.

14. Gester V. The microscopy in dental medicine: gadget or necessity? Rev Belge Med Dent (1984) 2004; 59: $62-76$.

15. Shanelec D A. Current trends in soft tissue. J Calif Dent Assoc 1991; 19: 57-60.

16. Sheiham A. Minimal intervention in dental care. Med Princ Pract 2002; 11(Suppl 1): 2-6.

17. Zappa U, Smith B, Simona C, Graf H, Case D, Kim W. Root substance removal by scaling and root planing. J Periodontol 1991: 62: 750-754

18. Perrin P J, Hotz P. Das Operationsmikroskop in der zahnärtzlichen Praxis: minimalinvasive Füllungen. Schweiz Monatsschr Zahnmed 2002; 112: 723-732.

19. Strassler H E. Clinical Uses of Reciprocating Handpieces. Inside Dentistry 2006; 2.

20. Tibbetts LS, Shanelec D. Periodontal microsurgery. Dent Clin North Am 1998; 42: 339-359.

21. Tibbetts $L S$, Shanelec D A. A review of the principles and practice of periodontal microsurgery. Tex Dent J 2007: 124: 188-204.

22. Velvart $P$, Peters $C I$. Soft tissue management in endodontic surgery. J Endod 2005; 31: 4-16.

23. Velvart $\mathrm{P}$, Ebner-Zimmermann U, Ebner J P. Comparison of long-term papilla healing following sulcular full thickness flap and papilla base flap in endodontic surgery. Int Endod J 2004; 37: 687-693.

24. Burkhardt R, Lang N P. Coverage of localized gingival recessions: comparison of micro-and macrosurgical techniques. J Clin Periodontol 2005; 32: 287-293.

25. Van Hattam A J. A model for the study of epithelial migratin in wound healing. Virchows Arch B Cell
Pathol Incl Mol Pathol 1979; 30: 221-230.

26. Francetti $L$, Del Fabbro M, Testori $T$, Weinstein

$R$ L. Periodontal microsurgery: report of 16 cases consecutively treated by the free rotated papilla autograft technique combined with the coronally advanced flap. Int J Periodontics Restorative Dent 2004: 24: 272-279.

27. Nordland W P, Sandhu H S, Perio C. Microsurgical technique for augmentation of the interdental papilla: three case reports. Int J Periodontics Restorative Dent 2008; 28: 543-549.

28. Shanelec D A. Anterior esthetic implants: microsurgical placement in extraction sockets with immediate plovisionals. J Calif Dent Assoc 2005; 33: $233-240$.

29. Cairo F, Carnevale G, Billi M, Prato G P. Fibre retention and papilla preservation technique in the treatment of infrabony defects: a microsurgical approach. Int J Periodontics Restorative Dent 2008; 28: 257-263.

30. Cortellini P, Tonetti M S. Microsurgical approach to periodontal regeneration. Initial evaluation in a case cohort. J Periodonto/ 2001; 72: 559-569.

31. Francetti L, Del Fabbro M, Calace S, Testori $T$, Weinstein R L. Microsurgical treatment of gingival recession: a controlled clinical study. Int $J$ Periodontics Restorative Dent 2005; 25: 181-188.

32. Association CODAAD. Standards for advanced speciality education programs in endodontics. Implementation Date: January 1st, 1998, Chicago.

33. Nordland W P, Tarnow D P. A classification system for loss of papillary height. J Periodontol 1998; 69: 1124-1126.

34. Shapiro A. Regeneration of interdental papillae using periodic curettage. Int J Periodontics Restorative Dent 1985; 5: 26-33. 